\title{
Peri-Operative Hearing Impairment
}

\author{
Vinodkumar Singh ${ }^{1}$, Ayush Sinha ${ }^{2}$, Rashmi Madan ${ }^{3}$ \\ ${ }^{1}$ Department of Anaesthetics, West Suffolk Hospital NHS Foundation Trust, Bury St Edmunds, UK; ${ }^{2}$ Department of Anaesthetics, \\ Guy's and St Thomas NHS Foundation Trust, London, UK; ${ }^{3}$ Department of Anaesthetics, Queen Elizabeth Hospital NHS Trust, \\ King's Lynn, UK. \\ Email: drvinbing@gmail.com
}

Received June 21 ${ }^{\text {st }}$, 2012; revised July 22 ${ }^{\text {nd }}, 2012$; accepted August $14^{\text {th }}, 2012$

\begin{abstract}
Peri-operative hearing impairment is a poorly reported morbidity following any type of anaesthesia. We report a case where the patient developed temporary hearing impairment after a gynaecological surgery. This recovered in a couple of week time. The causes of such hearing impairment in various types of anaesthesia are discussed.
\end{abstract}

Keywords: Hearing Impairment; Laryngeal Mask Airway; Nitrous Oxide

\section{Introduction}

Peri-operative hearing impairment is a poorly reported and distressing morbidity related to anaesthesia. It could occur following virtually any type of anaesthetic technique. There are 52 reported cases following general anaesthesia in literature [1]. The incidence of hearing loss after spinal anaesthesia is more common and can be as high as $50 \%$ [2]. The true incidence, however, is unknown, owing both to lack of reporting and subclinical presentation, which goes unnoticed unless audiometry is performed [3]. We report a case of bilateral hearing impairment following a minor gynaecological procedure in our day care unit.

\section{Case Report}

A previously well 54-year-old woman had hysteroscopy and insertion of mirena coil under general anaesthesia. After establishing intravenous access and standard noninvasive monitoring, she received $2 \mathrm{mg}$ midazolam and 75 mcg fentanyl. Anaesthesia was induced with $200 \mathrm{mg}$ propofol. After attaining adequate depth of anaesthesia, a size 4 laryngeal mask airway was introduced to maintain the airway. Anaesthesia was maintained with a mixture of oxygen and nitrous oxide (50:50) and isoflurane to a Minimum Alveolar Concentration (MAC) of 1.0. Patient breathed spontaneously through a Humphrey ADE circuit throughout the procedure. The procedure lasted fifteen minutes and her cardio-respiratory parameters remained stable throughout the procedure. She did not receive any ototoxic medications intra-operatively. The laryngeal mask airway was removed in the recovery room. The postoperative period was uneventful and she was discharged home after ninety minutes. Soon after her discharge from the hospital, the patient started experiencing difficulty in hearing in both the ears, more so on the left side. It gradually worsened overnight without throat pain or otalgia. There was no vertigo or otorrhoea either. Her General Practitioner saw her the next day with a normal systemic, ear and throat examination. She had bilateral sensori-neural hearing impairment on tuning fork test. On the basis of normal appearance of tympanic membrane, uvula, and soft palate; a decision was made to treat her conservatively with a provision to refer her to otolaryngologist in the event of deterioration. The hearing loss started resolving by the end of first week, followed by complete recovery by the end of second week.

\section{Discussion}

The exact cause of peri-operative hearing loss is often difficult to elucidate. However, several aetiologies have been proposed. Table 1 lists a few common causes of sudden hearing loss or impairment. Changes in cerebrospinal fluid pressure, middle ear pressures, damage to inner ear microcirculation, and ototoxic medications are few of the proposed mechanisms [2]. When cardiopulmonary bypass is employed, the hearing loss if often unilateral and particulate emboli are considered as the most likely cause [4]. Changes in the cerebrospinal fluid pressure are more common after a spinal anaesthetic. This is because the acute changes in the CSF pressure are immediately transmitted through the cochlear aqueduct to the perilymph, thus disrupting the position of the hair cells leading to hearing impairment [5]. During a general anaesthetic, changes in middle ear pressure can result from vigorous mask ventilation, blocked Eustachian tube 
Table 1. Some causes of sudden hearing impairment.

$$
\begin{aligned}
& \text { 1. Direct trauma } \\
& \text { 2. Surgical injury } \\
& \text { 3. Noise and barotraumas } \\
& \text { 4. Drugs and anaesthetic agents } \\
& \text { 5. Meniere's disease } \\
& \text { 6. Acoustic neuromas } \\
& \text { 7. Stroke and multiple sclerosis }
\end{aligned}
$$

or intra-operative use of nitrous oxide $\left(\mathrm{N}_{2} \mathrm{O}\right) . \mathrm{N}_{2} \mathrm{O}$ anaesthesia was shown in the late 1960s to be capable of causing oscillations of middle ear pressures that could result in tympanic perforation and cause hearing loss [6]. The degree of intra-tympanic pressure fluctuation and hence middle ear injury is a function of $\mathrm{N}_{2} \mathrm{O}$ concentration and its rate of change [7]. There is a need to build up the MAC rapidly in minor cases or procedures lasting for a short duration. In such cases, the likelihood of delivering higher concentration of nitrous oxide and consequent rapid rate of change could affect the middle ear pressures. This seems to be the most likely case in our patient that could have contributed to hearing loss. Also, during $\mathrm{N}_{2} \mathrm{O}$ elimination, Eustachian tube obstruction can result in significant negative middle ear pressure and tympanic perforation [8]. Uvular oedema and subsequent blocked Eustachian tube can also result in hearing loss after prolonged intubation or the use of oral or nasopharyngeal airways [9]. A laryngeal mask airway (LMA) was used in our patient for a very short duration and throat examination on the following day did not reveal any oedema or trauma to the uvula or posterior nasal space. Furthermore, any evidence to suggest a change in middle ear pressure due to the use of LMA is lacking [10]. Absence of vertigo and moderate hearing impairment on presentation were good prognostic factors in our patient's complete recovery by the end of second week.

Elucidation of exact aetiology of this rare anaesthetic morbidity would help the anaesthetists avoid factors leading to peri-operative hearing loss. In our case, nitrous oxide was the most likely factor that could have contributed to hearing loss. This case will help to fill the paucity of literature on peri-operative hearing loss and may prompt the clinicians to think before using $\mathrm{N}_{2} \mathrm{O}$ routinely for anaesthetising patients.

\section{REFERENCES}

[1] J. Punj, R. Pandey and V. Darlong, "Sensorineural Hearing Loss after General Anaesthesia: 52 Cases Reported Until Now,” Anaesthesia, Vol. 64, No. 2, 2009, p. 226.

[2] J. Sprung, D. L. Bourke, M. G. Contreras, M. E. Warner and J. Findlay, "Perioperative Hearing Impairment," Anaesthesiology, Vol. 98, No. 1, 2003, pp. 241-257.

[3] J. Fog, L. P. Wang, A. Sundberg and C. Mucchiano, "Hearing Loss after Spinal Anesthesia Is Related to Needle Size,” Anesthesia \& Analgesia, Vol. 70, No. 5, 1990, pp. 517-522.

[4] S. J. Millen, R. J. Toohill and R. H. Lehman, "Sudden Sensorineural Hearing Loss: Operative Complication in Non-Otologic Surgery,” Laryngoscope, Vol. 92, No. 6, 1982, pp. 613-617.

[5] A. Walsted, G. Salomon and K. S. Olsen, "Low-Frequency Hearing Loss after Spinal Anesthesia: Perilymphatic Hypotonia?” Scandinavian Audiology, Vol. 20, No. 4, 1991, pp. 211-215.

[6] L. Perreault, N. Normandin, L. Plamondon, R. Blain, P. Rousseau, M. Girard and G. Forget, "Middle Ear Pressure Variations during Nitrous Oxide and Oxygen Anaesthesia," Canadian Anaesthetists Society Journal, Vol. 29, No. 5, 1982, pp. 428-434.

[7] A. Man, S. Segal and S. Ezra, "Ear Injury Caused by Elevated Intratympanic Pressure during General Anaesthesia," Acta Anaesthesiologica Scandinavica, Vol. 24, No. 3, 1980, pp. 224-226.

[8] K. A. Thomsen, K. Terkildsen and I. Arnfred, "Middle Ear Pressure Variations during Anesthesia," Archives of Otolaryngology, Vol. 82, No. 6, 1965, pp. 609-611.

[9] G. L. Jones, R. M. Redfern and D. C. Jerwood, "Deafness -A Complication of Nasopharyngeal Airways," Anaesthesia and Intensive Care, Vol. 30, No. 6, 2002, pp. 807808.

[10] M. Hohlrieder, C. Keller, J. Brimacombe, S. Eschertzhuber, G. Luckner, I. Abraham and A. von Goedecke, "Middle Ear Pressure Changes during Anesthesia with or without Nitrous Oxide Are Similar among Airway Devices,” Anesthesia \& Analgesia, Vol. 102, No. 1, 2006, pp. 319-321. 